CARNETS DE Carnets de géographes

GÉOGRAPHES.

$10 \mid 2017$

Pour une réflexion collective sur l'enseignement de la géographie à l'Université

\title{
Entretien avec François Louveaux
}

François Louveaux

\section{(2) OpenEdition}

Journals

Édition électronique

URL : http://journals.openedition.org/cdg/1126

DOI : $10.4000 /$ cdg. 1126

ISSN : 2107-7266

Éditeur

UMR 245 - CESSMA

Référence électronique

François Louveaux, «Entretien avec François Louveaux », Carnets de géographes [En ligne], 10 | 2017, mis en ligne le 30 septembre 2017, consulté le 14 septembre 2020. URL : http://

journals.openedition.org/cdg/1126

Ce document a été généré automatiquement le 14 septembre 2020.

\section{(c) (1) () $\Theta$}

La revue Carnets de géographes est mise à disposition selon les termes de la Licence Creative Commons Attribution - Pas d'Utilisation Commerciale - Pas de Modification 4.0 International. 


\title{
Entretien avec François Louveaux
}

\author{
François Louveaux
}

\section{NOTE DE L'ÉDITEUR}

Inspecteur général de l'éducation nationale, François Louveaux a été interviewé par Marie Morelle et Jean Gardin le 13 septembre 2016, à l'Université Paris 1 où enseignent ces deux Maîtres de Conférences, dans les murs de l'Institut de géographie, rue Saint Jacques dans le quartier latin. Membre extérieur du conseil de gestion de l'UFR de géographie de cette université, François Louveaux a longtemps été professeur de géographie en classe préparatoire aux grandes écoles, au prestigieux lycée Henri IV, c'est-à-dire à une portée de flèche de là. Il a été pendant trois ans chargé par le Ministère de l'Enseignement Supérieur et de la Recherche d'aider à la mise en place des ESPE (Ecoles Supérieures du Professorat et de l'Education, succession des Instituts de Formation Universitaire des Maîtres, ou IUFM, elles-mêmes descendantes des Ecoles Normales). Il a aussi participé à la mise en place en région Auvergne Rhône Alpes d'un Institut Carnot de l'Education expérimental, destiné à faire travailler ensemble monde universitaire et monde scolaire pour des améliorations pédagogiques appuyées sur les apports de la recherche. Pour ces différentes raisons, il est fortement concerné par les questions pédagogiques telles qu'elles se posent à l'interface de l'enseignement secondaire et supérieur et il est bien au fait des questions de sélection sociale liées aux choix d'orientations et aux localisations géographiques.

\section{NOTE DE L'AUTEUR}

Entretien remanié le 8 mai 2017

\section{JG, MM : Merci d'avoir accepté notre invitation ...}

François Louveaux: Merci à vous pour votre invitation, Il est essentiel que monde scolaire et monde universitaire apprennent à se connaître, à travailler ensemble. Cela ne va pas de soi, entre un monde scolaire dont le fonctionnement reste largement 
hiérarchique et vertical et un monde universitaire globalement horizontal. J'ai pris une pleine conscience de cet enjeu avec l'accompagnement des ESPE. Les uns attendaient du Ministère qu'il impose une solution unique, valable partout, mettant en avant l'unicité du système de formation ; les autres considéraient l'intervention du Ministère comme insupportable, prônaient l'initiative de composantes universitaires autonomes, diverses et responsables. Le pari des ESPE, leur originalité est de faire travailler ensemble, en co construction, ces deux mondes. Il importe de la même façon d'assurer la meilleure transition de l'élève à l'étudiant. Pour cela le monde scolaire a besoin des universitaires, nous avons besoin de vous, nous avons besoin de travailler ensemble pour les élèves des collèges et des lycées...

JG, MM : Quand vous dites « on a besoin de vous», enseignants du supérieur, vous pensez à solliciter la recherche universitaire, pas la pédagogie?

F.L. : Dissocier recherche et pédagogie me semble un peu dangereux et m'incite à un détour plus large. Il y a dans le monde scolaire un long débat sur la place des disciplines scolaires dans l'organisation et la conception l'enseignement. Ce débat est vif. Certains pensent que l'organisation en disciplines est une des causes de l'échec de trop d'élèves et qu'il faut en conséquence envisager de façon très différente l'organisation de l'enseignement. Ce débat se noue aussi autour des compétences lesquelles ne sont pas l'apanage d'une seule discipline mais en convoquent plusieurs. Dans la réforme du collège on a vu l'importance prise par les débats autour de la place, de la nature, du rôle des enseignements pratiques interdisciplinaires (EPI). Cette réforme a d'ailleurs sans doute contribué à faire bouger un peu les lignes. Quoi qu'il en soit les disciplines fondent encore largement aujourd'hui l'identité professionnelle des enseignants. A mon sens, il faut partir de là, tout en sachant que le métier d'enseignant nécessite d'être redéfini dans un monde du numérique, de l'individualisation, de la globalisation. Le supposé modèle ancien, celui d'un enseignant seul détenteur du savoir devant des élèves qui ne savent rien, n'est plus tenable. Reste à construire, collectivement et cela vous implique, l'image de ce nouveau métier. Dans les débats sur les apports du numérique, on évoque parfois des enseignants presse-boutons délivrant des contenus tous prêts, rédigés par des universitaires, et qui n'auraient plus ensuite qu'à vérifier les résultats ou plutôt les acquis des élèves en appuyant sur un autre bouton. Nous n'en sommes pas là. Le métier d'enseignant est pour moi un métier d'intellectuel, celui d'un concepteur, qui travaille en équipe et en réseaux, lesquels doivent faire dialoguer enseignants du monde scolaire et universitaire. La dimension disciplinaire reste importante, des liens étroits avec l'université essentiels. J'ai été professeur en classes préparatoires mais j'ai aussi beaucoup participé à la préparation à l'agrégation interne à Poitiers. J'ai pu constater chez les collègues un profond souci de mise à jour scientifique. Nous avions mis en place des rencontres annuelles, entre universitaires, formateurs et professeurs, en alternance avec l'université de Poitiers et celle de la Rochelle. Elles ont connu un grand succès, tant auprès des professeurs du secondaire ravis de retrouver «leur " faculté, qu'auprès d'universitaires heureux d'échanger avec leurs collègues. Il n'y a de doute ni sur l'intérêt, ni sur les bénéfices de telles rencontres, reste sans doute à les organiser autrement, à rendre ces échanges habituels et systématiques, les réseaux offrant des possibilités décuplées.

Pour finir sur une note plus personnelle encore, j'ai participé ces dernières années au jury du prix de thèse du CNFG, une formidable opportunité pour moi de rester en 
contact avec la recherche qui se fait. Je suis frappé par la vitalité de la discipline que révèle la diversité de thèmes et des approches. La liste des thèses retenues est un bel outil pour montrer à des élèves ce que peut être la géographie. Je lis aussi des analyses qui seraient très précieuses à des enseignants du secondaire - je pense à une thèse de 2016 sur la Centrafrique. Voilà encore un chantier à ouvrir entre nous...

JG, MM : Nous venons de parler de l'agrégation interne, mais à part cela, quelles sont les autres formes de formation continue?

F.L.: Vous évoquez là le grand chantier à venir. Les débats sur la refondation de l'école en 2012 ont mis en avant une formule qui existait déjà mais est devenue un symbole : "enseigner est un métier qui s'apprend ». Ce succès est un peu paradoxal d'ailleurs car la formule relève de l'évidence, mais elle marquait la prise conscience qu'il fallait adopter une autre approche de la profession. Enseigner n'est pas un sacerdoce, même si la conscience du rôle social de ce métier est un facteur essentiel d'attractivité. Enseigner ce n'est pas seulement maitriser des connaissances et les transmettre, même si c'est la première qualité professionnelle. Enseigner suppose de partager des gestes professionnels, une culture et des valeurs où que l'on enseigne. Ce métier ne s'apprend pas une fois pour toute à 22 ans, il exige une formation continue ou même, pour reprendre l'expression employée par le comité de suivi de la réforme de la formation des enseignants, une formation tout au long de la vie. Les ESPE ont été créés pour assurer cette professionnalisation, qui est confiée à l'Université mais suppose un partenariat même une co-construction avec le monde scolaire. L'ESPE est à la fois une composante universitaire et un projet commun aux partenaires d'une académie, universitaires, scolaires et associations. Cela conduit entre autres à former des formateurs qui soient parfaitement à l'aise dans les deux univers scolaires et universitaire. Moins de quatre années après la création des ESPE, seul le volet de la formation initiale a été abordé. Le vaste chantier d'une offre académique de formation co-construite entre ESPE, universités, rectorat et partenaires académiques est devant nous. C'est aussi essentiel que délicat, il y a des formes nouvelles de formation à inventer, hybrides non seulement par la combinaison de formation en ligne et en présentiel, mais aussi par la combinaison entre offre institutionnelle, démarche individuelle et de groupes, formation nationale, académique et locale en établissements ou bassins.

JG, MM : Retournons la question : en quoi les universitaires ont-ils besoin du secondaire?

F.L.: Dans notre système éducatif on a l'habitude de raisonner en termes d'opposition, de rupture plus que de continuité et de complémentarité. Il y a le primaire puis le secondaire- collège puis lycée- et de l'autre côté de la «barrière » du baccalauréat, le supérieur, l'université. Cette vision est totalement dépassée quand la très grande majorité d'une classe d'âge fait des études supérieures, mais les représentations ont la vie dure. J'ajoute aussi qu'il fut un temps pas si lointain où les universitaires les plus reconnus s'investissaient dans les programmes du primaire comme du secondaire. A mon sens, aller travailler avec et pour les enseignants du secondaire et aussi du primaire devrait faire partie des missions de certains universitaires, à des moments de leur carrière. Il ne s'agit bien sûr pas d'imaginer des heures obligatoires à aller donner au rectorat. Je suis sûr qu'on a besoin de vous... Mais est-ce que vous ça peut vous apporter quelque chose? Je pense que c'est toujours intéressant d'enseigner à des publics différents, qui n'ont pas les mêmes 
références, les mêmes acquis, les mêmes attentes. Cela conduit à réorganiser, repenser son travail et ainsi à l'enrichir, mieux le maîtriser.

JG, MM: Ce n'était pas le sens de la question: on vient chercher les enseignants chercheurs pour leur volet recherche, mais pas pour leur volet enseignant, alors, est ce que la pédagogie à l'université existe?

F.L.: Je ne répondrai pas sur la pédagogie à l'Université, un domaine que je ne connais pas. Ce dont je suis sûr c'est que vos étudiants sont les ex élèves du lycée et qu'ils ne changent pas du tout au tout une fois le baccalauréat obtenu. Nous travaillons tous pour construire progressivement des adultes responsables et engagés. Dans l'enseignement secondaire on a pris progressivement conscience que l'enseignement frontal, le seul cours magistral ne convenait plus à la totalité des élèves, qu'il fallait aussi réfléchir à d'autres méthodes, qu'il fallait échanger, développer le travail en équipe tant des élèves que des enseignants. Cette prise de conscience est passée du primaire au collège, puis aujourd'hui au lycée... cela ne s'arrêtera pas à une pseudo barrière du baccalauréat. Comme toujours dans notre système éducatif cela crée des tensions, des inquiétudes et l'on dénonce successivement la primarisation du collège, la transformation du lycée en collège, la secondarisation du supérieur... Comme toujours aussi on finit par ne plus voir que les difficultés, les échecs, par exemple les échecs en première année du supérieur. Or, Je crois que ce qui a été acquis ( presque) pour le secondaire, l'idée qu'enseigner est un métier avec la discipline pour noyau fondamental, mais que ce noyau est insuffisant car on travaille avec des individus et des groupes qui arrivent avec des passés différents, des désirs différents, des projets différents, dans un environnement profondément transformé par le numérique et une société qui se cherche. Cet effort pédagogique ne s'arrêtera pas aux portes de l'Université. D'ailleurs, si on gratte un peu, on se rend compte qu'il y a des dizaines d'expériences qui se font au quotidien dans les universités, que ces questions pédagogiques sont débattues. Cela n'est pas lié à des injonctions, mais a ce qui fait la richesse de notre système éducatif, primaire, secondaire comme supérieur, l'engagement des acteurs de terrain. Les ESPE ont aussi dans leur périmètre l'aide à la professionnalisation du métier d'enseignant du supérieur.

JG, MM : donc les ESPE peuvent jeter un coup d'œil sur la pédagogie à l'Université ?

F.L. : Les ESPE sont des composantes universitaires de plein exercice, pas en dehors de l'Université, même si leur originalité est d'être en position d'interface entre scolaire et universitaire. La question illustre bien une réalité : rien ne se fera de manière automatique, encore moins autoritaire, cela passe par du dialogue, de la cogestion, la coresponsabilité, pas par l'obligation, de l'intrusion, ou de l'injonction : cela ne marche pas. Je crois que les structures sont là pour faciliter, permettre l'action et l'initiative des acteurs, pas pour figer ou imposer, être des fins en soi : ce temps-là est terminé. La réforme de la formation engagée en 2013 illustre concrètement la volonté de faire se rencontrer, via les ESPE, scolaire et universitaire. Les lauréats des concours de recrutement deviennent des fonctionnaires stagiaires qui ont à la fois un demi service d'enseignement en responsabilité et l'obligation de réussir un $\mathrm{M} 2$ incluant la rédaction et la soutenance d'un mémoire de master. Cette double obligation suppose une formation originale fondée sur une alternance intégrative, c'est-à-dire une formation qui articule les réalités du métier éprouvées sur le terrain et la formation universitaire. Ce n'est pas simple à mettre en place, c'est 
encore très imparfait, mais on a là un exemple concret de la volonté d'articuler universitaire et scolaire, qui suppose d'ailleurs aujourd'hui pour les fonctionnaires stagiaires un double tutorat, universitaire et scolaire. De même, les nouvelles épreuves du CAPES introduisent une dimension professionnelle au-delà de la seule maitrise scientifique mais en étroite relation avec elle, c'est du moins ce qui est souhaité. Voilà comment peut se construire ce dialogue entre universitaire et scolaire. On se parle entre intellectuels quand même.

JG, MM : Vous avez fait allusion à la démocratisation de l'Université. Vous avez dit que les problèmes qui se posent au secondaire se poseront dans le supérieur, vous faisiez allusion à cela?

F.L. : Aujourd'hui beaucoup d'étudiants, ex lycéens, ne sont plus adaptés au modèle classique de transmission et d'acquisition des connaissances et compétences, ne sont plus en connivence intellectuelle avec le modèle d'enseignement qui a formé les formateurs que nous sommes. A partir de ce constat, ou bien on ne retient que ceux qui sont les plus adaptables, ou bien on cherche, sans renier les exigences, à trouver des formes pédagogiques qui permettent au plus grand nombre de mieux réussir.

JG, MM: Dans l'entretien que vous avez donné pour la revue Echogeo il y a quelques années ${ }^{1}$, vous vouliez préparer les lycéens à l'université, mais il semble que restait à définir ce qu'était cette université : cela sous-entendait que les enseignants du supérieur puissent prendre en charge ces nouveaux étudiants. Donc cela marche dans les deux sens : préparer les lycéens à l'université, préparer l'université à ces étudiants ?

F.L. : Dans les programmes politiques, dans les discours de responsables de la CPU, on parle de plus en plus de réformer le baccalauréat et de mieux ajuster les cursus pré et post baccalauréat. D'un côté un examen final avec un nombre réduit d'épreuves; de l'autre des pré requis, le fait par exemple d'avoir suivi tel ou tel enseignement, qui conditionnent l'entrée dans telle ou telle filière, dans telle ou telle université. On retrouve bien la logique de la continuité, mais vue à partir de l'autre côté : les universités détermineraient une part de ce qui doit être acquis dans le secondaire afin de garantir une bonne poursuite d'études et des succès. Ajoutons que les débats les plus vifs portent moins sur les filières générales que sur bacs techno et pro, lesquels accueillent massivement les élèves issus des milieux les moins favorisés. La question est donc complexe et éminemment politique, à un moment où, en outre, le sujet de l'orientation ou sélection revient en force dans le paysage universitaire.

Au-delà de ces choix politiques, une telle analyse obligerait à revoir plus ou moins profondément l'organisation et la nature des enseignements au lycée. La géographie serait particulièrement concernée. On pourrait imaginer que des renforcements en statistiques, une initiation aux SIG, la lecture d'images, la réalisation cartes et croquis puissent être valorisés pour entrer dans une licence de géographie. Cette liste est sommaire et incomplète, tout cela serait justement à discuter entre universitaires, professeurs du secondaire, inspecteurs et responsables des programmes. On peut supposer alors la mise en place d'un enseignement optionnel - un peu sur le modèle de ce qui s'était fait en terminale scientifique l'année où l'histoire-géographie n'y étaient plus obligatoires. On mesure là une des difficultés qu'il y aurait à résoudre. Au lycée histoire et géographie sont associées, ce qui n'est pas du tout systématique à l'université. Elles sont conçues comme des disciples de formation générale, présentes dans toutes les filières, destinées à donner des repères, des connaissances, mais aussi l'habitude d'examiner de façon critique textes et images, de débattre, avec pour objectif d'aider des lycéens à devenir des citoyens actifs dans un monde complexe. On 
est bien dans une optique de formation générale. Même si l'Université- à ce que je crois- évolue vers des licences moins mono disciplinaires, les logiques sont et resteront différentes. Là encore, ce qu'il faut imaginer c'est un dialogue, une construction qui exige que les acteurs se parlent, se connaissent. Tout est à construire, avec des méthodes à inventer et une exigence d'ouverture de respect et de confiance.

JG, MM : La question de qui accueillir et pour quelles finalités ; les étudiants sont-ils bien à leur place? A quoi les forme l'université? Un métier? Des compétences? Ce sont les mêmes questions que dans le secondaire finalement.

F.L. : J'ai l'impression que l'on retrouve en effet des débats communs, sur les moyens de faire réussir tous les étudiants ou sur l'équilibre entre une formation générale et des compétences professionnelles, propres à préparer à un métier, ce qui renvoie aux finalités même de l'enseignement. Au ministère, la direction générale qui suit l'université a désormais pour titre «enseignement supérieur et insertion professionnelle ». Là encore les oppositions peuvent paraitre surprenantes. On a du mal en France à penser autre chose qu'une voie unique, un cursus unique, une forme unique de réussite et de l'excellence. Tous les bacheliers auraient-ils vocation à faire une thèse ? On a du mal à penser la diversité autrement qu'en terme de hiérarchie, d'inégale réussite ou dignité, et c'est une question qui dépasse largement notre sujet. On a du mal aussi à imaginer des cursus qui ne soient pas linéaires et à penser que tout ne se fait pas dans la seule formation initiale, entre 20 et 25 ans. On a du mal à penser en termes de continuité, de progressivité, à imaginer des bifurcations. Dans une thèse récente Leila Frouillou s'intéresse à la ségrégation universitaire. Elle met en évidence un phénomène très intéressant qui est la plasticité du système universitaire : des étudiants échouent mais reprennent ensuite des études, souvent après avoir travaillé, et finalement réussissent dans une autre voie. Dans un ordre d'idée un peu comparable, dans le recrutement d'enseignants, en particulier professeurs des écoles, on constate de plus en plus de secondes carrières: des collègues choisissent l'enseignement après avoir exercé un autre métier, ce qui oblige d'ailleurs à repenser nos méthodes de formation.

JG, MM : Oui, et cela marche dans les deux sens : d'un côté on peut considérer comme échec quelque chose de très enrichissant sur le temps long; et a contrario, avec un suivi post-diplôme limité à plus six mois ou un an après le diplôme, on peut avoir une information très pauvre sur l'emploi de quelqu'un que l'on catégorise comme «ayant réussi » dans la mesure où il a un emploi.

F.L.: Il faudrait connaitre les trajectoires, il y aurait tout un travail d'accompagnement à assumer. Il est difficile aujourd'hui, faute d'un suivi des étudiants, d'un suivi de cohortes, d'avoir autre chose que des impressions.

JG, MM : Peut-on parler un peu de votre parcours?

F.L. : Moi c'est du très classique! J'étais un élève studieux, obéissant. Je suis allé en classes préparatoires, puis j'ai eu la chance de réussir le concours d'entrée à l'Ecole Normale Supérieure de Saint Cloud, j'ai obtenu l'agrégation et un DEA. J'ai eu une expérience très utile et formatrice : l'enseignement dans des classes technologiques dans deux lycées de Rouen. Les cours avaient souvent lieu de $16 \mathrm{~h}$ à $18 \mathrm{~h}$, après les heures de mathématiques et d'informatique. Cela a été très formateur, je n'y étais pas du tout préparé, heureusement que j'ai eu d'excellents collègues pour m'aider et d'abord me soutenir, me rassurer... J'ai eu la chance d'être très vite nommé en classes préparatoires, mais je n'ai jamais oublié mes élèves de techno. J'ai terminé à côté, à 
Henri IV. Puis ensuite, parcours classique toujours, j'ai postulé à l'Inspection Générale où j'ai participé entre autres à des missions sur les classes préparatoires, sur la formation des enseignants, entre autres la masterisation de 2010-2012, ce qui m'a conduit logiquement à être chargé pendant les trois dernières années de participer à l'accompagnement de la mise en place des ESPE.

JG, MM : Pour revenir plus précisément sur les classes préparatoires, quand on parlait de la diversité des parcours, qu'un échec puisse être une réussite, des questions de court et long terme, quelle place donneriez-vous aux «prépas » aujourd'hui par rapport aux premières années de licence à l'université ?

F.L. : Avec cette question, on reste bien dans ce que nous évoquons depuis le début : la nécessite de penser en termes de continuité et de complémentarité plus qu'en opposition, le décalage entre la réalité des évolutions et des perceptions aussi vives que dépassées, souvent. Historiquement, il y a eu des classes préparatoires car il y a eu des grandes écoles et des grandes écoles parce qu'à un moment de son histoire, à la charnière du XIXème siècle, l'Université n'a pas voulu assumer des formations professionnelles qu'il a donc fallu créer en dehors. Aujourd'hui l'Université assure des formations professionnelles et cela se développe. Pour avoir eu l'honneur d'être choisi pour siéger parmi les personnalités extérieures au conseil de gestion de l'UFR de géographie de Paris 1, j'ai découvert à quel point les stages et les commandes des entreprises et des organisations apportent à la formation mais aussi aux moyens de l'UFR, non sans débats d'ailleurs. Le doctorat est désormais, en France comme partout dans le monde, le symbole de l'excellence. Grandes écoles et universités travaillent de plus en plus ensemble sur les segments doctorat et master, participent à des constructions élargies, certes non sans difficultés et réticence. Ce qui se fait en aval se fera en amont avec les CPGE, dont les étudiants sont d'ailleurs désormais, symbole fort, inscrits à l'université. Je n'ai rien à dire sur les premiers cycles universitaires que je ne connais pas. J'ai bien « "visité » (c'est le terme officiel) à l'université des PRAG (professeurs agrégés affectés dans le supérieur) qui souhaitaient postuler en CPGE, mais cela relève de l'anecdote. Il faut donc penser en termes de complémentarité CPGE et Université. Dans les CPGE littéraires d'ailleurs l'immense majorité des étudiants rejoint l'université après les deux, trois années de prépa. Cette liaison est beaucoup moins directe dans les autres CPGE, mais on a déjà évoqué les rapprochements avec les grandes écoles et on constate aussi que dans les écoles de management des étudiants choisissent de faire leur année de césure (une année obligatoire hors cursus) dans des universités. Pour faire court, les CPGE sont pour leurs étudiants le début de leur formation supérieure. Elles sont bien adaptées à certains étudiants, moins ou pas à d'autres, à cause de leur originalité - la pluri disciplinarité - et d'une ingénierie pédagogique fondée sur un suivi très régulier, ponctué par la répétition d'exercices comme la dissertation, exercices que l'on retrouve ensuite dans les concours de recrutement $d$ 'enseignants, ce qui est pour les étudiants des CPGE qui choisissent cette voie un atout. A cela s'ajoute bien sûr une sélection à l'entrée qui augmente les chances de réussite, même s'il ne faut pas sousestimer pour ces étudiants aussi, le "gap » entre ce qui est demandé au lycée et ce qui est nécessaire pour réussir des études supérieures. Ce système est cohérent, bien financé, il a des résultats tangibles. Ceci dit le système des $\mathrm{CP}$ est contesté parce qu'elles seraient coupées de la recherche, ne favoriseraient pas le travail en équipe, l'imagination, reposeraient plus sur la répétition de solutions connues que sur la capacité à en inventer de nouvelles dans un monde de l'imprévu. A cela s'ajoute une 
vive critique de la sélection, de la charge de travail, du coût et du caractère socialement sélectif des CPGE qui seraient, de fait, réservées aux enfants issus des milieux favorisés et aux enfants ... d'enseignants, bref à ceux qui connaissent les codes scolaires, l'importance du diplôme, ont les moyens d'apporter des compléments de formation à leurs enfants. Tout cela mériterait de longs développements et surtout beaucoup de nuances. Le débat est pollué par des attitudes parfois fermées. Le mépris qu'affichaient certains professeurs de CPGE visà-vis des premiers cycles universitaires a fort heureusement pratiquement disparu, même si subsistent quelques réfractaires, pas toujours chez les professeurs les plus anciens. Du côté des détracteurs des CPGE, il y aurait aussi à dire, parfois. Simplement convenir que les CPGE sont bien adaptées à des étudiants qui sont prêts à fournir une bonne quantité de travail, se sentent bien dans ce système extraordinairement guidant et qui souvent hésitent sur leur orientation future. Les premiers cycles universitaires sont eux aussi des voies de réussite et d'excellence. Tous se retrouveront ensuite dans le second cycle universitaire.

JG, MM : oui, parce que la licence est disciplinaire mais l'université demande aussi une autonomie individuelle. De leur côté, les CP encadrent, vous les disiez guidantes, mais en même temps, elles encadrent.

F.L. : Cet encadrement a ses avantages, mais il faut aussi développer l'autonomie, le sens du travail, de l'analyse, de la réflexion en commun, savoir aussi quitter le pluri disciplinaire et le général pour des études plus pointues, approfondies, techniques parfois. Notons au passage les vertus ici du numérique : désormais les étudiants et les préparateurs des CPGE ont un accès facile aux articles de revues scientifiques, cela constitue un gain important pour la formation, tandis que le numérique favorise le travail en commun. Pour autant les grandes écoles constatent que la première année s'accompagne parfois d'un certain spleen, d'effets de décompression parfois spectaculaires, voire inquiétants. Cela doit interroger les grandes écoles, beaucoup remanient leur première année, mais aussi bien sûr les CPGE et les concours. Encore une fois les progrès viendront d'un dialogue, d'une meilleure connaissance réciproque. Les étudiants de CPGE qui entrent à l'Université se sentent un peu perdus au début, sont mal à l'aise avec l'autonomie, découvrent aussi les contraintes administratives. Cela mériterait sans doute aussi une réflexion sur la L3. Ne pourrait-on pas imaginer, dans certaines voies, de mixer les apports méthodologiques des $\mathrm{CP}$ et de l'université? Si un jour on décidait de placer en fin de L3 les concours de recrutement d'enseignants cette question ne serait plus seulement rhétorique.

JG, MM : Dans ce cas, avec un concours en fin de L3, il n'y aurait plus que des CP qui deviendraient profs, c'est certain, parce que quelqu'un qui a fait sa L1 et sa L2 ici à Tolbiac, en L3 (le site Pierre Mendès France de l'UP1), il ne va pas forcément réussir le concours.

F.L. : Ce serait bien sûr totalement inacceptable! Pourquoi ne pas imaginer alors en L3 un parcours préparation aux concours insistant sur la préparation des exercices écrits et oraux, auxquels sans doute des professeurs de CPGE, fort de leur expérience des concours, pourraient utilement participer? Symétriquement d'ailleurs l'intervention en CPGE d'enseignants chercheurs est une richesse. Dans cette hypothèse- nous pourrions voir très vite si elle est une simple fiction - la définition et le contenu des épreuves des concours seraient bien sûr décisifs et ne peuvent s'imaginer en porte à faux avec ce qui se fait à l'université. Le principe du concours n'implique pas des formes et des contenus intangibles.

JG, MM : I faut quand même qu'on insiste un peu sur les premiers cycles universitaires. C'est un problème qu'on connait mal comme enseignants parce que pour l'essentiel les collègues du supérieur ne les ont pas connus de l'intérieur, comme étudiants. C'est un 
problème abondamment traité par les sociologues de l'éducation, et on passe dessus toujours aussi rapidement.

F.L.: Ça me rappelle un échange avec Agnès Van Zanten autour des multiples opérations d'ouverture sociale dans les CPGE. Beaucoup d'enseignants de CPGE ont le sentiment de pouvoir encore, par les CPGE, faire réussir des étudiants d'origine modeste. C'est vrai et il ne faut surtout pas le négliger. Ce qui est vrai aussi c'est que, particulièrement depuis une vingtaine d'année, on s'aperçoit qu'en France la réussite scolaire est très liées à l'origine sociale voire territoriale des élèves. Les études internationales le démontrent. C'est pour beaucoup d'enseignants, parfaitement insupportable. Cela pose de façon évidente la question d'un modèle scolaire qui est nettement influencé par les CPGE. En parallèle, le fait que les enseignants soient de moins en moins issus des milieux d'où viennent leurs élèves, et pas seulement à l'université, pose des défis nouveaux, en particulier vis-à-vis élèves issus des milieux les plus défavorisés. Ces questions sont graves et méritent mieux que des affirmations péremptoires et des solutions « simples ».

$J G$ : Ça fait penser à une réflexion que je me fais souvent quand je prépare au CAPES. J'ai un public charmant, intéressé, avec un objectif clair, et moi du coup, j'ai moi aussi un objectif clair.

F.L. : Les concours, c'est un système stimulus-réponse parfait : les étudiants savent pourquoi ils sont là, et on sait à quoi les préparer. C'est la même chose en CP. Et ce système stimulus-réponse n'est pour autant pas une machine à abrutir. Plus les étudiants avancent dans leur préparation, plus ils se posent des questions intéressantes. Ce qui me semble le plus dommageable dans le système français, c'est que l'on imagine un seul modèle de réussite, largement fondé sur des concours et des diplômes obtenus à vingt ans. Comme si seule comptait la réussite universitaire, comme si tout se décidait à vingt ans, sans possibilité d'ascension, d'évolution, de reprise de formation ensuite. C'est dramatique. Et c'est typically french.

$J G, M M$ : Mais quels sont les points de comparaison internationaux?

F.L. : Les systèmes éducatifs ne sont pas les mêmes partout, parce que ni l'histoire, ni les sociétés ne sont les mêmes. C'est pour ça que les comparaisons internationales sont toujours un peu délicates. Par exemple, s'interroger sur le caractère socialement hyper sélectif des études les plus prestigieuses en Grande Bretagne n'a aucun sens, littéralement la question ne se pose pas. Aux Etats-Unis, l'affirmative action s'explique par le souci, nouveau, de faire une place aux minorités. Chez nous les questions d'ouverture sociale ont un fort écho, car elles renvoient à une certaine conception de notre modèle républicain. Ailleurs la réussite serait considérée comme le fruit du seul mérite individuel...

JG, MM : Oui mais à part l'affirmative action, quelle est la réelle connaissance que nous avons de systèmes éducatifs étrangers, justement?

F.L. : Pas beaucoup, mais les évaluations internationales sont désormais considérées comme des repères majeurs. On leur reprochait de méconnaitre les spécificités françaises, d'être mal adaptées à nos choix d'enseignement. Aujourd'hui on utilise souvent ces évaluations pour culpabiliser les enseignants en leur disant que la France est de plus en plus mal classée, que «son niveau baisse». Je doute que la culpabilisation soit le meilleur moyen de mobiliser les enseignants, de les aider à réfléchir à leurs pratiques, de les aider à les améliorer.... 
JG, MM: Du côté du premier cycle universitaire on avait eu à un certain moment des financements, avec le plan Licence, dans les années 2010-2012, et on en a profité pour essayer un encadrement un peu plus personnalisé, avec des sorties de terrain... mais quel est le bilan?

F.L. : On ne tire jamais le bilan des expériences et les évolutions suivantes ne sont pas pensées en fonction d'évaluations des précédentes. C'est une question de structures : qui, au-delà des inspections générales peut évaluer ? Plusieurs réponses nouvelles s'esquissent, en particulier l'appel à des chercheurs. C'est aussi le problème des politiques. Les questions d'éducation sont très vives en France et c'est une chance. En revanche, cela pousse les responsables politiques à vouloir montrer qu'ils agissent et à chercher des résultats rapides, au rythme de leur chronologie politique. Or, dans le domaine éducatif, il faut du temps : la Ministre souligne que l'on ne mesurera les effets de la réforme du collège de 2016 que dans les évaluations PISA de 2020 ou 2025 (Programme international pour le suivi des acquis des élèves, mené par l'OCDE, comparant les capacités acquises à 15 ans). Je pense qu'un certain nombre de systèmes sont à bout de souffle et que cela commence à faire consensus. Il faut apprendre à travailler de manière différente, décloisonnée, sans promouvoir un système unique. La réforme des ESPE de même demandera du temps, on ne décrète pas une co-construction entre université et monde scolaire. Cela se construit à partir des habitudes de travail en commun qui s'instaurent sur le terrain.

JG, MM : Au sujet de ce lien ESPE- Universités, as-tu fait partie de comités de spécialistes pour le recrutement de maîtres de conférences en ESPE?

F.L. : Non, car je ne suis pas enseignant-chercheur. Cette réponse est logique, mais interroge aussi peut-être un peu lorsque l'on parle de l'ESPE comme d 'un projet commun?

JG, MM : Parce qu'on a parfois un peu l'impression que postuler à l'ESPE reste un second choix.

F.L.: Je n'ai pas d'éléments de réponse, mais tant que la formation ne sera pas clairement affichée par les universités comme un axe stratégique, prioritaire, on peut craindre qu'il n'en soit ainsi. Les ESPE sont des composantes universitaires, mais elles n'ont pas toutes une proportion d'enseignants chercheurs comparable à celle des autres composantes. La transformation de postes de PRAG en postes EC a des conséquences financières non négligeable, les obligations de service variant du simple au double. Ce chantier est ouvert pour les ESPE, il demandera du temps des moyens et donc une ferme volonté politique.

JG, MM : Mais on en revient toujours à la difficulté à mettre ça en place dans le premier cycle, pas en Master.

F.L. : Il est de plus en plus clair qu'il faut développer une " préprofessionnalisation » avant le Master pour ceux qui se destinent aux métiers de l'enseignement, en particulier qu'ils puissent aller voir ce qui se passe concrètement dans des établissements scolaires. On peut aussi ajouter un volet social, en permettant à des étudiants de poursuivre leurs études en étant rémunérés pour quelques heures de travail dans un établissement scolaire. Cela a été expérimenté ces dernières années, avec de vrais succès mais aussi des aléas. Cette piste devrait se préciser et s'amplifier, on peut le souhaiter en tous cas.

JG, MM : On a quand même l'impression qu'il y a un hiatus : à l'université, on a affaire à des gens qui sont spécialistes de tel ou tel sujet sur le plan de la recherche. Du coup, quand il s'agit de pédagogie, on sent comme un refus de se voir enseigner à enseigner. Tel ou tel 
spécialiste a toujours tendance à penser que sur tel sujet ultra précis qu'il est à peu près le seul à maitriser, personne ne peut lui donner de leçons de pédagogie.

F.L. : En France, on a fait le choix exigeant du modèle d'enseignant-chercheur qui suppose des qualités différentes mais complémentaires. Lorsque j'enseignais en CPGE, j'avais pour habitude de demander à un enseignant chercheur de faire une intervention sur le programme du concours, en général en fin de préparation, à un moment où les étudiants avaient une connaissance de base de la question. Cette intervention était toujours très riche pour eux et ils mesuraient bien, entre autres à la précision du vocabulaire, à la maîtrise fine des concepts, aux choix des exemples, la distance qu'il y a entre un généraliste et un spécialiste. On conçoit que sur certains sujets très pointus, seuls les spécialistes puissent s'adresser à des spécialistes, mais on est là dans le domaine de la recherche. Ce qui me semblerait important et logique c'est de ne pas réserver les interventions de chercheurs au post licence et de n'avoir en premier cycle que des généralistes non chercheurs. Pour autant, intervenir en premier cycle suppose aujourd'hui de savoir là aussi s'adapter à des étudiants qui n'ont plus le profil classiquement attendu... et là le supérieur rejoint le secondaire...

J.G. : J'avais encore une question sur le rôle du numérique. Tu y es venu rapidement de toi même dans cet entretien. On peut y revenir une seconde?

F.L. : J'ai un peu l'impression que l'on présente parfois le numérique la solution miracle qui va résoudre tous les problèmes, ceux de l'enseignement en particulier. C'est un travers fréquent chez nous de croire qu'il y aurait une solution unique. Dans le domaine éducatif, le numérique propose en effet beaucoup de pistes prometteuses : l'accès facile à des données, la possibilité d'individualisation des parcours et des enseignements, à la fois l'autoformation et la possibilité de travail en groupe et plus encore en collectifs. De grandes sociétés sont prêtes à fabriquer de très bons programmes clés en main, à fournir des ressources pour enseigner, pour évaluer et cela représente des marchés considérables. L'hybride s'impose, en particulier pour la formation continue et l'autoformation. Pour autant, je ne crois pas à un enseignant qui serait ne quelque sorte un simple guide, accompagnateur, metteur en œuvre de ressources numériques. Je ne crois pas qu'il faille orienter la formation des enseignants vers ce modèle-là. L'utilisation des ressources numériques est un atout, mais, pour moi, au service d'une conception qui reste le domaine de l'enseignant.

J.G. : Mais tu as quand même présenté le rapport au numérique comme un des moteurs essentiels de la transformation du rapport au professeur

F.L. : Le numérique condamne, s'il en était encore besoin, une vision caricaturale d'un enseignant qui sait face à des élèves qui ne savent pas et aujourd'hui les élèves vérifient en temps réel les affirmations du professeur, en particulier les statistiques, les dates. Tout ou presque est disponible sur le web, encore faut-il savoir chercher, évaluer la pertinence de ce qui est offert et construire à partir de cela. Une information n'est pas une connaissance surtout qu'il y a des informations incomplètes voire fausses. Une utilisation efficace du numérique suppose sans doute en amont de comprendre le fonctionnement et la logique de la machine - on commence à s'en rendre compte - mais aussi les réflexes critiques, les connaissances de base et la culture qui permettent de trier efficacement les informations comme les analyses disponibles. Si le professeur n'a pas le monopole des connaissances, il lui reste à aider l'étudiant à se construire un esprit ouvert, curieux et critique et à lui apprendre comment bâtir un raisonnement, une argumentation, une réfutation, mener une controverse, choisir entre les argumentations que sera capable de fournir 
une machine. Il n'est plus possible d'enseigner sans le numérique, enseigner avec le numérique ne fait pas disparaitre le professeur, il lui donne un rôle autre, plus grand encore sans doute.

Pardon d'avoir été aussi long et sans doute parfois trop allusif, mais je suis un passionné des sujets éducatifs. Je ne suis pas le seul et on peut même dire que presque chaque français à ses avis sur la question, tant l'Ecole est liée à la République et au projet de société qu'elle sous-tend. Cela donne une vraie place aux sujets d'éducation et c'est une chance. Cela rend aussi les débats complexes, passionnés, crée de fortes impatiences et donc complique beaucoup des évolutions qui ont besoin de temps et de continuité. Mais il me semble qu'aujourd'hui bien des dossiers sont mûrs pour engager des transformations indispensables. Ces libres propos sont strictement personnels et n'engage en rien, bien sûr, l'Inspection générale

\section{NOTES}

1. François Louveaux, «La diversité de la géographie en CPGE : une faiblesse ou un atout? », EchoGéo [Online], $10 \mid 2009$, Online since 06 November 2009, connection on 12 June 2017. URL : http://echogeo.revues.org/11461 ; DOI : 10.4000/echogeo.11461

\section{INDEX}

Thèmes : Carnets de débats 